Research article

urn:1sid:zoobank.org:pub:BF7A05CC-65A0-41E6-91E9-2C10FD5EC2DF

\title{
Two new species of Willowsia (Collembola: Entomobryidae) from Yunnan Province, China
}

\author{
Rui $\mathrm{CHAI}^{1}$ \& Yitong $\mathrm{MA}^{2, *}$ \\ ${ }^{1,2}$ School of Life Science, Nantong University, Nantong 226000, P.R. China. \\ ${ }^{*}$ Corresponding author: mayitong@ntu.edu.cn \\ ${ }^{1}$ Email: chairui2015@126.com \\ ${ }^{1}$ urn:lsid:zoobank.org:author:0702F470-4C20-4044-A29F-57A35D38FC29 \\ ${ }^{2}$ urn:1sid:zoobank.org:author:14F92F90-863A-46EB-B056-41EB3FA341A0
}

\begin{abstract}
We describe two new species of Willowsia: W. baoshanensis sp. nov. and W. zhaotongensis sp. nov. This is the first report of Willowsia from Yunnan Province, China. W. baoshanensis sp. nov. is mainly characterized by its chaetotaxy, $W$. zhaotongensis sp. nov. by its color pattern and chaetotaxy.
\end{abstract}

Keywords. Entomobryinae, taxonomy, chaetotaxy, scales.

Chai R. \& Yitong M. 2017. Two new species of Willowsia (Collembola: Entomobryidae) from Yunnan Province, China. European Journal of Taxonomy 311: 1-12. https://doi.org/10.5852/ejt.2017.311

\section{Introduction}

The genus Willowsia was erected by Shoebotham (1917) to accomodate the species Seira nigromaculata Lubbock, 1873 from England, mainly based on its pointed scales. Its main generic characters include mucro bidentation and the presence of a basal spine, eyes $8+8$, dental spine absent. Scale morphology and distribution are important in taxonomy. Scales may be absent on the antennae, the legs and the manubrium in some species and are always absent on dens.

Thirty species have been described from all over the world and their habitats are from arctic regions to tropical areas. Thirteen species were described or reported from China. Here, two new species from Yunnan, southwest China, are described and a key to the Chinese species of the genus is given.

\section{Material and methods}

Specimens were mounted under a coverslip in Marc André II solution and were subsequently studied with a Leica DM2500 microscope. Photographs were taken with a mounted Leica DFC300 FX digital camera and enhanced with Photoshop CS2 (Adobe Inc.). The nomenclature of the dorsal chaetotaxy of head and interocular chaetae is described following Jordana \& Baquero (2005), Szeptycki (1979) and Mari-Mutt (1986). Labial chaetae are designated following Gisin (1967). Tergal chaetae of the body are designated using the system of Szeptycki (1979). Morphology of scales is described following Zhang, Chen \& Deharveng (2011). 


\section{Abbreviations:}

Abd. $=$ abdominal segment

Ant. = antennal segment

Mac $=$ macrochaeta $(\mathrm{e})$

Th. = thoracic segment

\section{Results}

Class Collembola Lubbock, 1873

Order Entomobryomorpha Börner, 1913

Family Entomobryidae Tömösvary, 1882

Subfamily Entomobryinae Schäffer, 1896

Genus Willowsia Shoebotham, 1917

\section{Diagnosis}

Moderate size, usually 1-2 mm; eyes $8+8$; four segmented antennae; mucro bidentate and with a basal spine; dentes without spine; various scale types in different species and absent on dens.

Willowsia baoshanensis sp. nov. urn:1sid:zoobank.org:act:A334FCB0-054E-4527-BC0B-F61A322CD9DC

Figs 1-3; Table 1

\section{Diagnosis}

Eyepatches and lateral margin of Th. II and III with a little blue pigment; Abd. I with 4+4 mac; Abd. II usually with $4+4$ dorso-central mac; Abd. III with $2+2$ dorso-central and $3+3$ lateral mac; spinulate type scales present on Ant. I, head, terga, legs, ventral tube and ventral side of manubrium.

\section{Etymology}

The specific epithet refers to the type locality: Baoshan City.

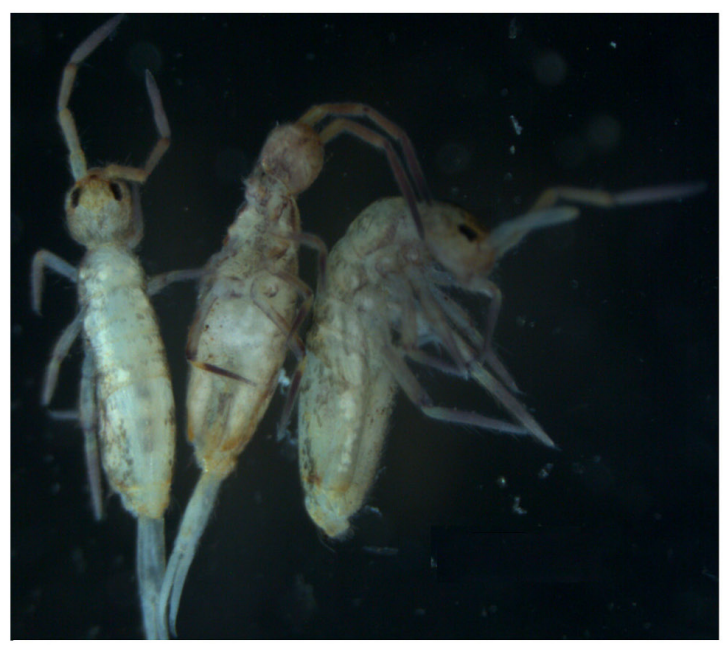

A

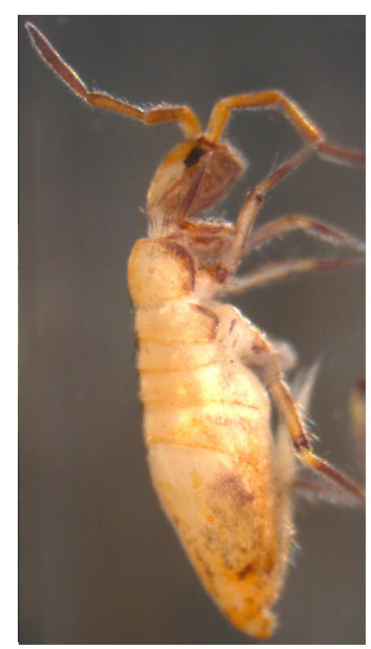

$\mathrm{B}$

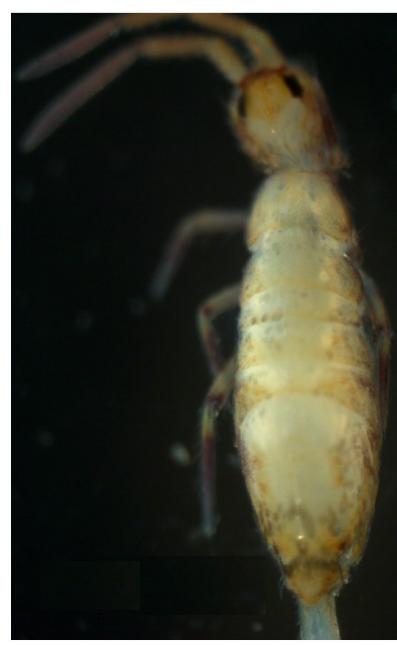

C

Fig. 1. Willowsia baoshanensis sp. nov. A-C. Habitus (A, C = paratypes; B = holotype) Scale bars: $500 \mu \mathrm{m}$. 
Table 1. Comparison of W. baoshanensis sp. nov., W. guangxiensis Shi \& Chen, 2004 and W. shi Pan, Zhang \& Chen, 2006.

\begin{tabular}{lccc}
\hline Characters & W. baoshanensis sp. nov. & W. guangxiensis & W. shi \\
\hline Labial triangle setae & $\mathrm{M}_{1} \mathrm{M}_{2} \mathrm{REL}_{1} \mathrm{~L}_{2}$ & $\mathrm{M}_{1} \mathrm{M}_{2} \mathrm{M}_{3} \mathrm{RRSEL}_{1} \mathrm{~L}_{2}$ & $\mathrm{MREL}_{1} \mathrm{~L}_{2}$ \\
Scales on legs & present & present & absent \\
Central macrochaetae on & $4(\mathrm{a} 2, \mathrm{~m} 3, \mathrm{~m} 3 \mathrm{e}, \mathrm{m} 3 \mathrm{ep}$, & $5(\mathrm{a} 2, \mathrm{a} 3, \mathrm{~m} 3 \mathrm{ep}$, & $5(\mathrm{a} 2, \mathrm{~m} 3 \mathrm{ea}, \mathrm{m} 3 \mathrm{ep}$, \\
Abd. II & rarely $5, \mathrm{a} 3 \mathrm{present})$ & $\mathrm{m} 3 \mathrm{e}, \mathrm{m} 3)$ & $\mathrm{m} 3 \mathrm{e}, \mathrm{m} 3)$ \\
Central macrochaetae on & $2(\mathrm{a} 2, \mathrm{~m} 3)$ & $2(\mathrm{a} 2, \mathrm{~m} 3)$ & $3(\mathrm{a} 2, \mathrm{a} 3, \mathrm{~m} 3)$ \\
Abd. III & $3(\mathrm{am} 6, \mathrm{pm} 6, \mathrm{p} 6)$ & $4(\mathrm{am} 6, \mathrm{pm} 6, \mathrm{p} 6, \mathrm{~m} 7)$ & $5(\mathrm{am} 6, \mathrm{pm} 6, \mathrm{p} 6, \mathrm{~m} 7, \mathrm{p} 7)$ \\
Lateral macrochaetae on & & & 4 \\
Abd. III & 4 & 3 & 4 \\
Ungual inner teeth & &
\end{tabular}

\section{Type material}

\section{Holotype}

CHINA: + , on slide, collected in Guanjia Village, Xinjie Town, Longyang District, Baoshan City, Yunnan Province, rotten leaves of bamboo, 10 Aug. 2014, Xinnan Jiang leg. (collection number: 1148, Nantong University).

\section{Paratypes}

CHINA: 5 $q$ on slides, 2 $q+$ in alcohol, same data as holotype.

\section{Description}

Colour pattern. Body length up to $1.9 \mathrm{~mm}$. Ground color pale yellow. Eyepatch dark blue. Ant. IV and tibiotarsus with blue pigment. Lateral margin of Th. II and III also with a little blue pigment (Fig. $1 \mathrm{~A}-\mathrm{C})$.

HEAD. Antenna $0.43-0.57$ times as long as body. Ratios of length of antennal segments I:II:III:IV = 1:1.6-2.0:1.6-2.4:2.5-4.1. Distal part of Ant. IV with many sensory setae, normal ciliate setae and apical bulb bilobed (Fig. 2A). Dorsal cephalic chaetotaxy with 6-7 antennal (An), 5 (4) median ( $\mathrm{M}_{1}-\mathrm{M}_{4}$, an additional seta between $\mathrm{M}_{2}$ and $\mathrm{M}_{3}$ rarely absent) and 8 sutural $\left(\mathrm{S}_{0}-\mathrm{S}_{5}, \mathrm{~S}_{4 \mathrm{i}}, \mathrm{S}_{5 \mathrm{i}}\right)$ mac. Interocular area with $\mathrm{p}, \mathrm{s}$, t setae. Eyes 8+8, G and $\mathrm{H}$ smaller (Fig. 2B). Labral setae as 4/5, 5, 4, all slender; prelabral setae ciliate, other smooth; distal margin of labrum with 4 papillae, each with 1 denticle (Fig. 2C). Lateral process of labial palp straight, as thick as normal setae, with tip not reaching apex of labial papilla (Fig. 2D). Labial triangle setae as in Fig. 2E, all finely ciliate.

Thorax. Dorsal macrochaetae shown as in Fig. 2F. Th. II with 2 (m1, m2) medio-median, 2 (m4, m4i) medio-lateral and 14-18 posterior mac on each side, 2 specialized setae (S-setae) on antero-lateral margin not clearly seen. Th. III with 13-15 median and 7 (m5, a6, a6i, p5, p6, m6, m6e) lateral mac on each side. Trochanteral organ with 26-30 smooth spiny setae (Fig. 2G). Unguis with 4 inner teeth, one pair located $0.38-0.39$ from base of inner edge of unguis, distal unpaired two respectively at $0.68-0.71$ and $0.84-0.89$ distance from base. Unguiculus acuminate and outer edge serrate. Tenent hair thick with clavate tip, almost equal length to inner side of unguis (Fig. 2H).

Abdomen. Abd. IV 4.2-8.0 times as long as Abd. III along dorsal midline. Dorsal mac shown in Figs 2I and 3A. Abd. I with 4 (m2, m3, m4, m4p) mac. Abd. II with 4 (a2, m3, m3e, m3ep) (rarely 5, a3 present), 1 (m5) lateral mac and 2 S-setae. Abd. III with 2 (a2, m3) dorso-central, 3 (am6, pm6, p6) 

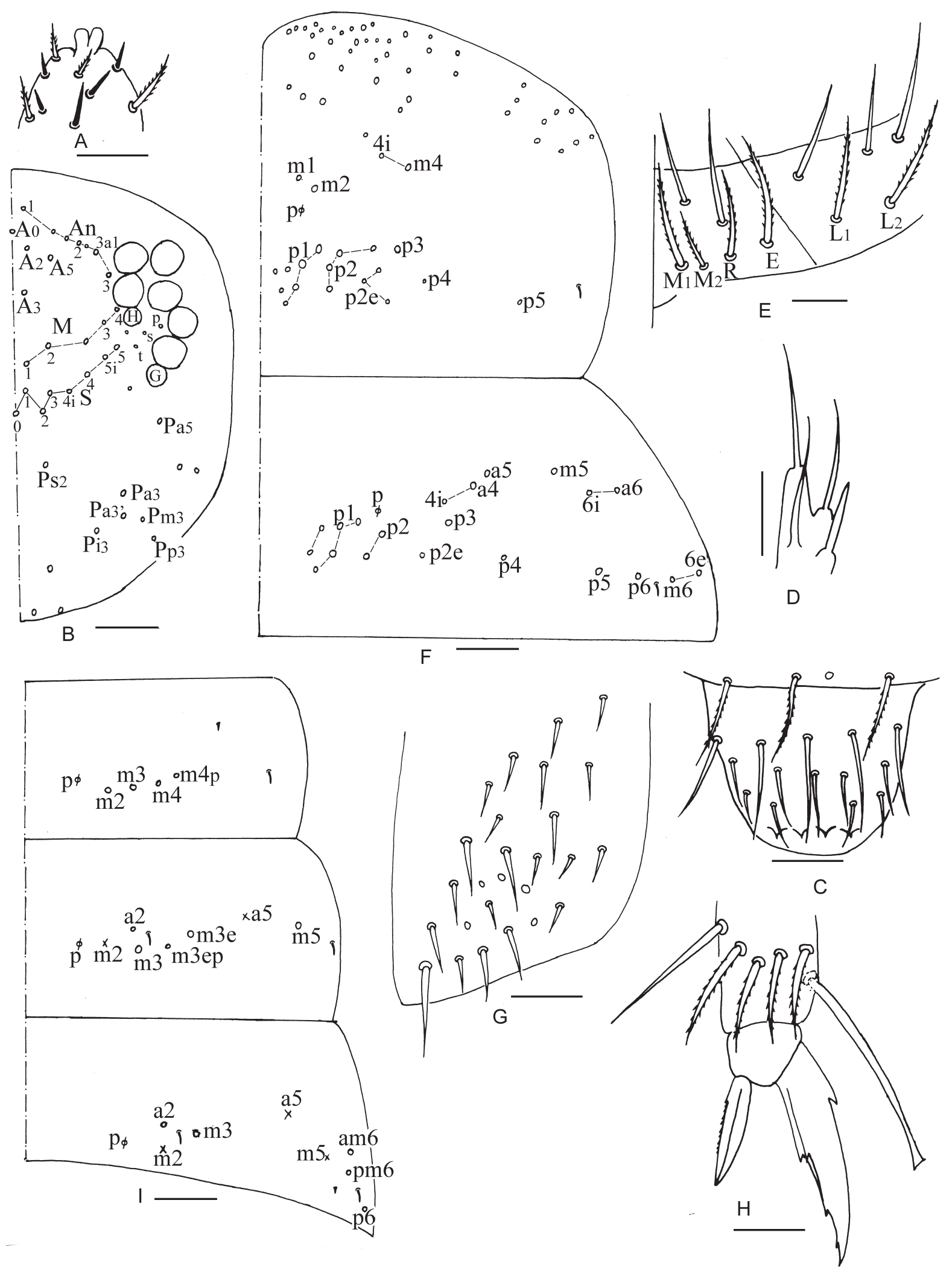

Fig. 2. Willowsia baoshanensis sp. nov. A. Apex of Ant. IV. B. Dorsal chaetotaxy of head. C. Labrum. D. Lateral process of labial palp. E. Labium. F. Chaetotaxy of Th. II-III. G. Trochanteral organ. H. Hind foot complex. I. Chaetotaxy of Abd. I-III. Scale bars: A, C-E, G-H $=15 \mu \mathrm{m} ; \mathrm{B}, \mathrm{F}, \mathrm{I}=50 \mu \mathrm{m}$. 

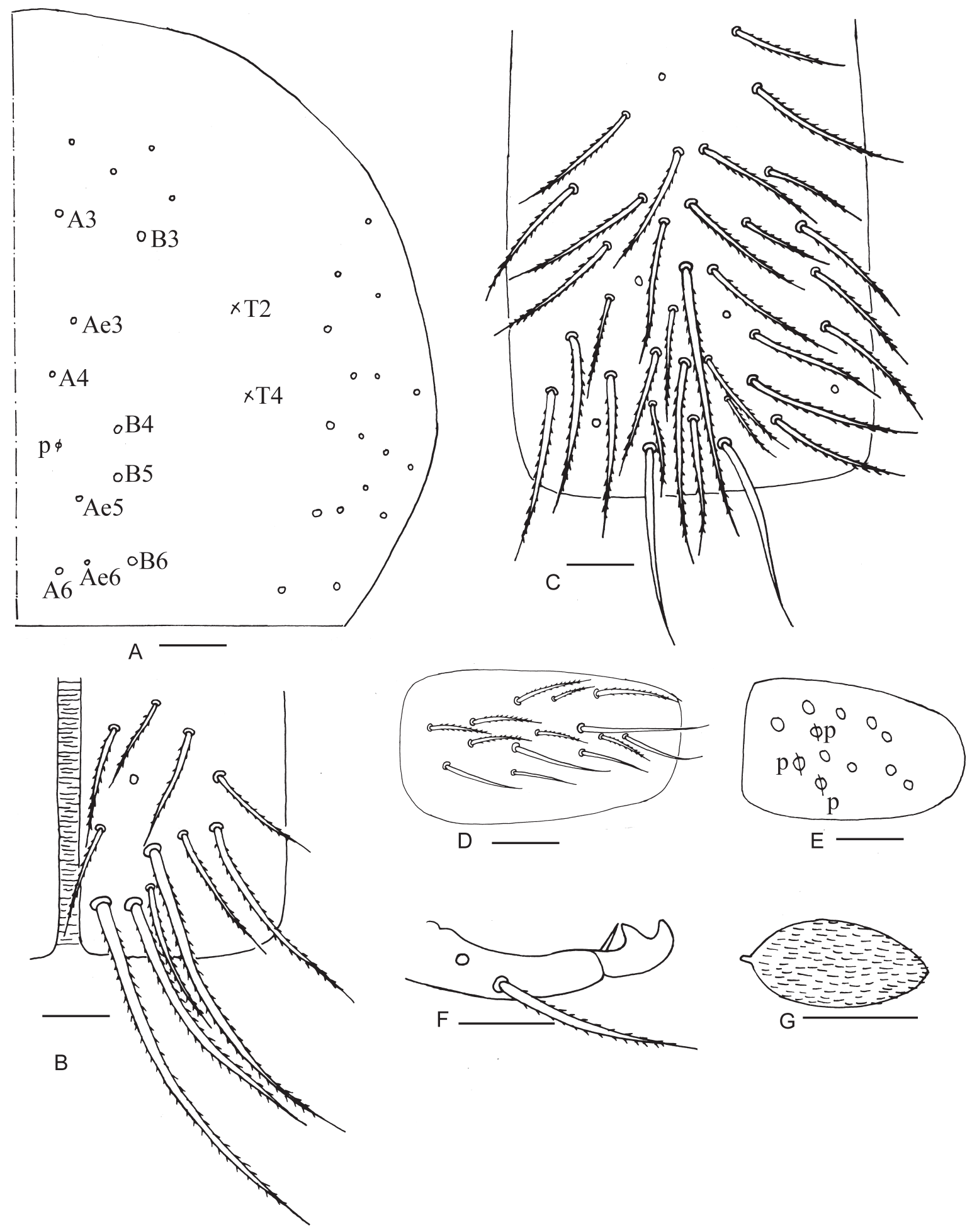

Fig. 3. Willowsia baoshanensis sp. nov. A. Chaetotaxy of Abd. IV. B. Anterior face of ventral tube. C. Posterior face of ventral tube. D. Lateral flap of ventral tube. E. Plaque of manubrium. F. Distal part of dens and mucro. G. Scale. Scale bars: $A=50 \mu \mathrm{m} ; \mathrm{B}-\mathrm{G}=15 \mu \mathrm{m}$. 
lateral mac and 3 S-setae (Fig. 2I). Abd. IV with 11-18 central (rarely 7, A3, A4, A6, B3, B4, B5, B6 always present) and 15-17 lateral mac as in Fig. 3A. Ventral tube anteriorly with 3 large and about 9 small ciliate setae (Fig. 3B), posteriorly with 2 apical smooth setae and about 36 ciliate setae of different sizes (Fig. 3C), laterally with 6 smooth and 8 ciliate setae of different sizes (Fig. 3D). Manubrial plaque with $8-9$ ciliate setae and 3 pseudopores on each side (Fig. 3E). Dens without spines, uncrenulated dens 1.5-1.7 times as long as mucro. Mucro bidentate, two teeth subequal and tip of basal spine reaching subapical tooth (Fig. 3F).

SCALES. All scales spinulate type and present on Ant. I, head, body, legs and ventral tube, ventral side of manubrium (Fig. 3G).

\section{Remarks}

The new species can be distinguished from other species of the genus in its color pattern. It is very similar to Chinese species W. guangxiensis Shi \& Chen, 2004 and W. shi Pan, Zhang \& Chen, 2006 in scales and chaetotaxy on Abd. I, but their color pattern, labial triangle setae, chaetotaxy on Abd. II and III and ungual inner teeth are different as shown in Table 1.

Willowsia zhaotongensis sp. nov.

urn:1sid:zoobank.org:act:C1D8D5E5-3BF2-4726-B875-2B729F0DB073

Figs 4-6; Table 2

\section{Diagnosis}

Dorsal part of Abd. II \& III and median and posterior parts of Abd. IV central with blue pigments; Abd. I usually with 4+4 mac; Abd. II with 4-5+4-5 dorso-central mac; Abd. III with 3+3 dorso-central and $5+5$ lateral mac; spinulate type scales present on Ant. I, head, body, legs, ventral tube and ventral side of manubrium.

\section{Etymology}

The specific epithet refers to the type locality: Zhaotong City.

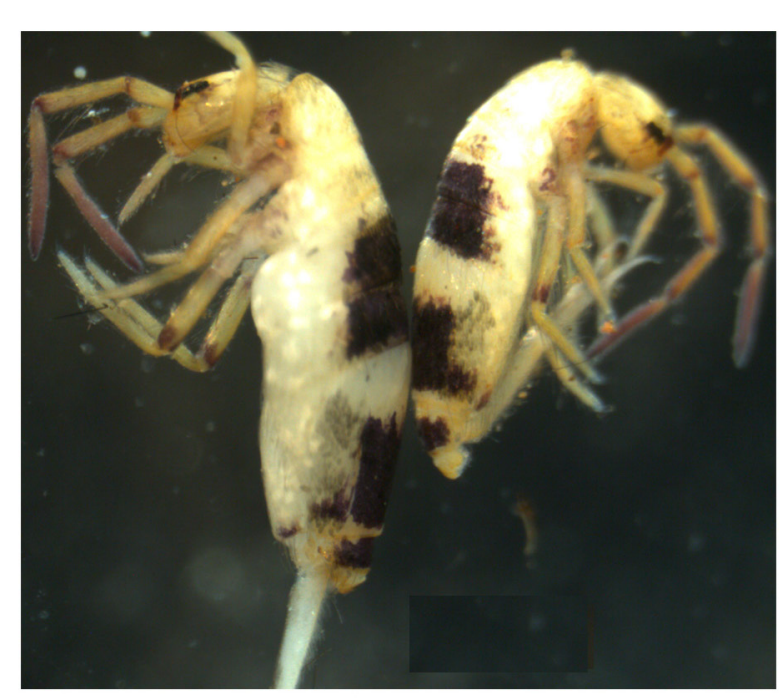

A

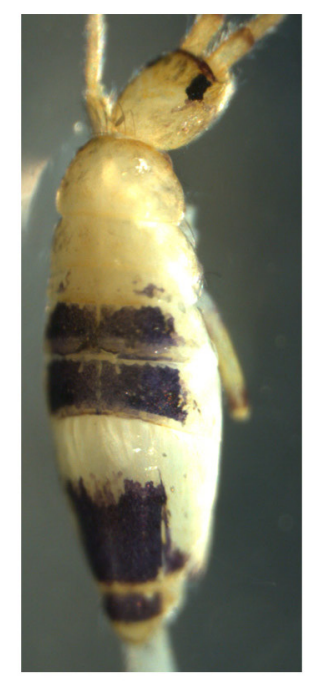

$\mathrm{B}$

Fig. 4. Willowsia zhaotongensis sp. nov. A-B. Habitus (A = paratype; B = holotype). Scale bars: $500 \mu \mathrm{m}$. 
Table 2. Comparison of $W$. zhaotongensis sp. nov. and W. bartkei Stach, 1965.

\begin{tabular}{lcc}
\hline Characters & W. zhaotongensis sp. nov. & W. bartkei \\
\hline a1, a2 \& a3 on Th. III & absent & present \\
m3ep on Abd. II & present & absent \\
a3 on Abd. III & present & absent \\
Ae2, Ae3 \& Ae6 on Abd. IV & present & absent \\
Scales on antennae and furcula & present & absent \\
Uncrenulated dens to mucro in length & $6-7$ & 4 \\
\hline
\end{tabular}

\section{Type material}

\section{Holotype}

CHINA: , on slide, collected in Yuanlong Village, Yongfeng Town, Zhaoyang District, Zhaotong City, Yunnan Province, rotten stems of maize, 1 Aug. 2014, Fudong Ding leg. (collection number 1149, Nantong University).

\section{Paratypes}

CHINA: $7 q+$ on slides, 4 $q$ in alcohol, same data as holotype.

\section{Description}

Colour Pattern. Body length up to $2.3 \mathrm{~mm}$. Eyepatch dark blue. Dorsal part of Abd. II \& III and median and posterior parts of Abd. IV centrally with blue pigment. Coxa and distal part of Ant. IV weakly pigmented (Fig. 4A-B).

HEAD. Antenna $0.45-0.56$ times as long as body. Ratios of length of antennal segments I:II:III:IV = 1:1.6-2.0:1.3-1.7:2.3-3.1. Distal part of Ant. IV with many sensory setae, normal ciliate setae and apical bulb biolobed (Fig. 5A). Dorsal cephalic chaetotaxy with 7 antennal (An), 4 median $\left(M_{1}-M_{4}\right)$ and 9 sutural $\left(\mathrm{S}_{0}-\mathrm{S}_{6}, \mathrm{~S}_{4 \mathrm{i}}, \mathrm{S}_{5 \mathrm{i}}\right)$ mac. Interocular area with $\mathrm{p}, \mathrm{s}$, t setae. Eyes $8+8, \mathrm{G}$ and $\mathrm{H}$ smaller (Fig. 5B). Labral setae as 4/5, 5, 4, all slender; prelabral setae ciliate, other smooth; distal margin of labrum with 4 papillae, each with 1 denticle (Fig. 5C). Lateral process of labial palp straight, as thick as normal setae, with tip not reaching apex of labial papilla (Fig. 5D). Labial triangle setae as in Fig. 5E, all finely ciliate.

THORAX. Dorsal macrochaetae shown as in Fig. 5F. Th. II with $2(\mathrm{~m} 1, \mathrm{~m} 2)$ (rarely 3, m2i present) mediomedian, $3(\mathrm{~m} 4, \mathrm{~m} 4 \mathrm{i}, \mathrm{m} 4 \mathrm{p})$ medio-lateral and 16-18 posterior mac on each side, 2 S-setae present on medio-lateral margin. Th. III with 16-17 median and 6 (a6, a6i, p5, p6, m6, m6e) lateral mac on each side. Trochanteral organ with 42-49 smooth spiny setae (Fig. 5G). Unguis with 4 inner teeth, one pair located $0.45-0.50$ from base of inner edge of unguis, distal unpaired two respectively at $0.70-0.74$ and 0.85-0.88 distance from base. Unguiculus acuminate and outer edge serrate. Tenent hair thick with clavate tip, slightly longer than inner side of unguis (Fig. 5H).

ABdomen. Abd. IV 2.8-5.0 times as long as Abd. III along dorsal midline. Dorsal mac shown in Figs 6A \& 6B. Abd. I with 4 (m2, m3, m4, m4p) (rarely 3, m4p absent) mac. Abd. II with 4-5 (a2, a3, m3, $\mathrm{m} 3 \mathrm{e}, \mathrm{m} 3 \mathrm{ep}, \mathrm{a} 3$ sometimes absent), 1(m5) lateral mac and 2 sensory chaetae. Abd. III with 3 (a2, a3, m3) dorso-central, 5 (am6, pm6, p6, m7a, p7) lateral mac and 3 sensory chaetae (Fig. 6A). Abd. IV with 10-12 central (A3, A4, A6, B3, B4, B5, B6 always present) and 17-20 lateral mac as in Fig. 6B. Ventral tube anteriorly with 3 large and 15-23 small ciliate setae (Fig. 6C), posteriorly with 2 apical smooth setae and about 35 ciliate setae of different sizes (Fig. 6D); lateral flap not clearly seen. Manubrial plaque with 


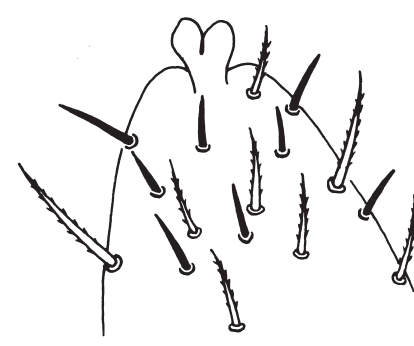

A
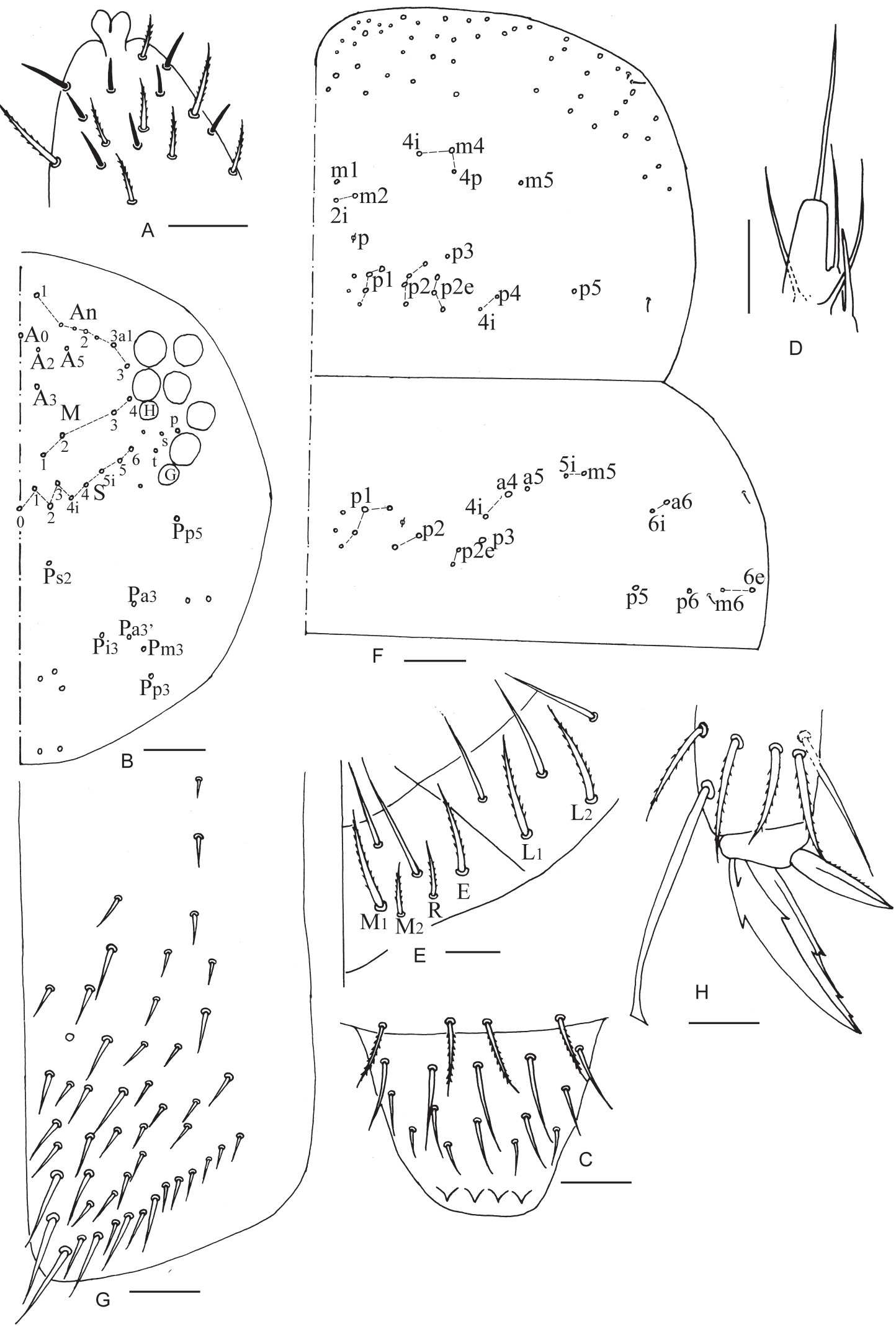

Fig. 5. Willowsia zhaotongensis sp. nov. A. Apex of Ant. IV. B. Dorsal chaetotaxy of head. C. Labrum. D. Lateral process of labial palp. E. Labium. F. Chaetotaxy of Th. II-III. G. Trochanteral organ. H. Hind foot complex. Scale bars: A, C-E, $\mathrm{G}-\mathrm{H}=15 \mu \mathrm{m} ; \mathrm{B}, \mathrm{F}=50 \mu \mathrm{m}$. 

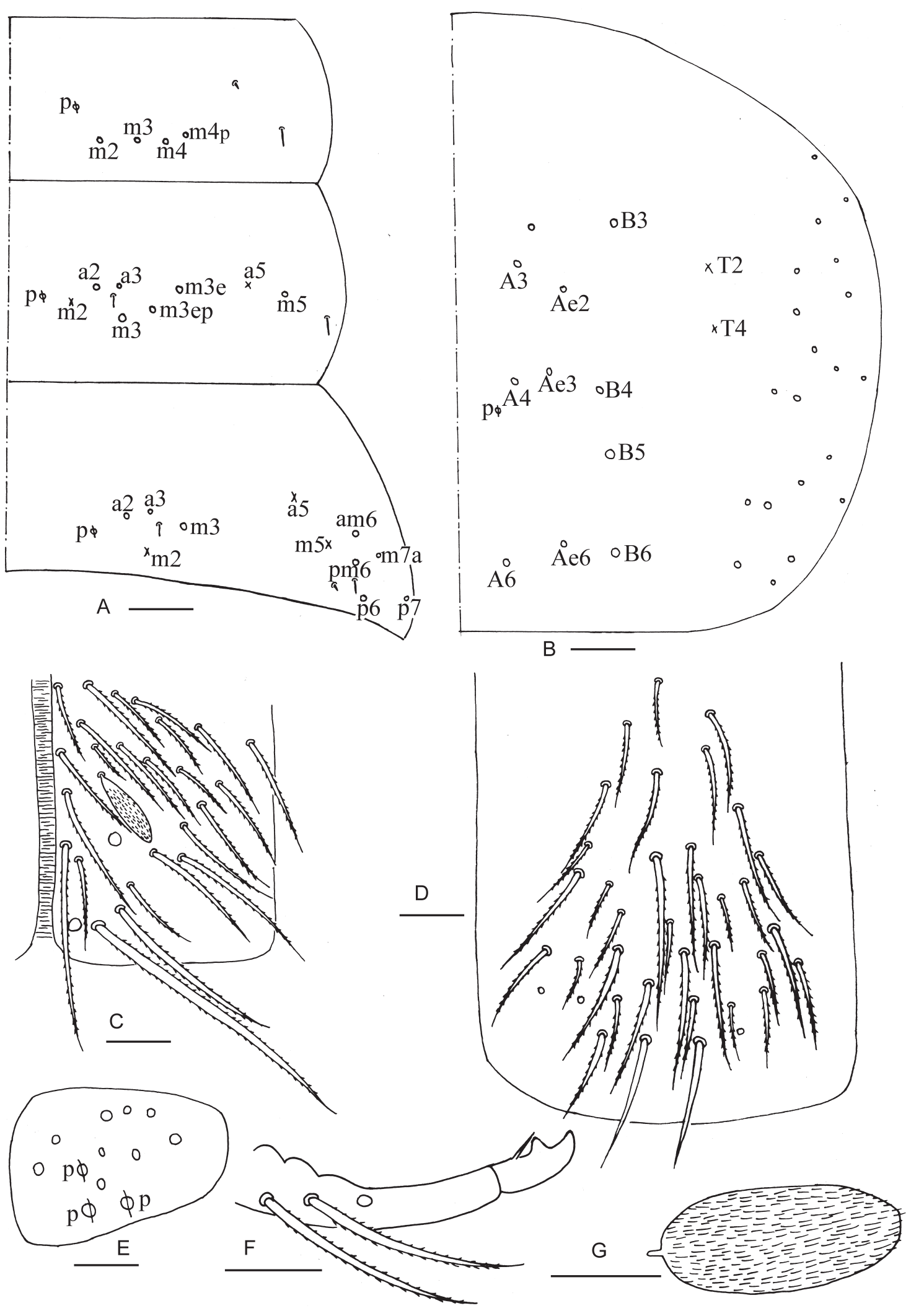

Fig. 6. Willowsia zhaotongensis sp. nov. A. Chaetotaxy of Abd. I-III. B. Chaetotaxy of Abd. IV. C. Anterior face of ventral tube. D. Posterior face of ventral tube. E. Plaque of manubrium. F. Distal part of dens and mucro. G. Scale. Scale bars: $A-B=50 \mu \mathrm{m} ; \mathrm{C}-\mathrm{G}=15 \mu \mathrm{m}$. 
8-11 ciliate setae and 3(4) pseudopores on each side (Fig. 6E). Dens without spines, uncrenulated dens 1.4-1.7 times as long as mucro. Mucro bidentate, two teeth subequal and tip of basal spine reaching subapical tooth (Fig. 6F).

SCALES. All scales spinulate type and present on Ant. I, head, body, legs and ventral tube, ventral side of manubrium (Fig. 6G).

\section{Remarks}

The new species can easily be distinguished from the other species of the genus by their color pattern, except for the Vietnamese species Willowsia bartkei Stach, 1965. Both new species have blue pigment on the dorsal part of Abd. II and III and on the median and posterior parts of Abd. IV centrally, but there is no blue pigment on Abd. I posteriorly and Abd. II and III laterally in the former, whereas pigment is present in the latter. The other differences, such as chaetotaxy and scales, are listed in Table 2.

\section{Key to the Chinese species of the genus Willowsia}

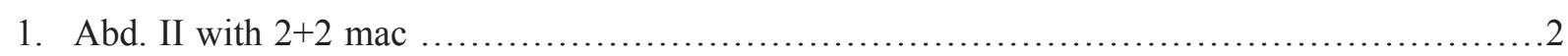

- Abd. II with at lest $3+3$ central mac ........................................................ 3

2. Abd. III with $1+1$ central mac .................................... formosana Denis, 1929

- Abd. III with $2+2$ central mac ...................................... jacobsoni (Börner, 1913)

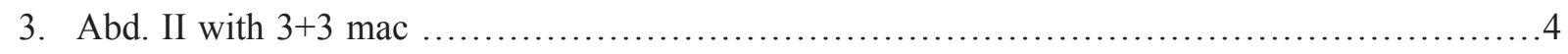

- Abd. II with at lest $4+4$ mac .............................................................. 11

4. Abd. I with $4+4$ mac ..................................W. qui Zhang, Chen \& Deharveng, 2011

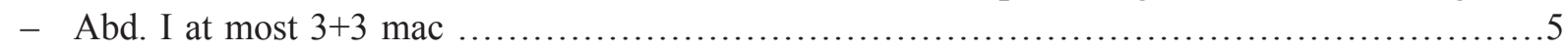

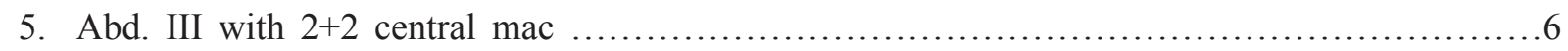

- Abd. III with $3+3$ central mac ..........................................................

6. Scales present on antennae ..................... guangdongensis Zhang, Xu \& Chen, 2007

- Scales absent on antennae ................................................................

7. Body scales of short rib type .................................. japonica (Folsom, 1897)

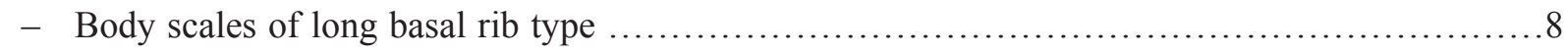

8. Abd. IV with $5+5$ central mac ............................. pseudobuskii Pan \& Zhang, 2016

- Abd. IV with $7+7$ central mac .............................. pseudoplatani Zhang \& Pan, 2016

9. Abd. II without blue transverse band .........................W. nigromaculata (Lubbock, 1873)

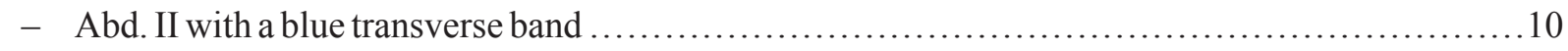

10. Metathorax blue pigmented, cephalic $\mathrm{S}_{0}$ absent ..................... similis Pan \& Zhang, 2016

- Metathorax unpigmented, cephalic $\mathrm{S}_{0}$ present ......................W. fascia Zhang \& Pan, 2016

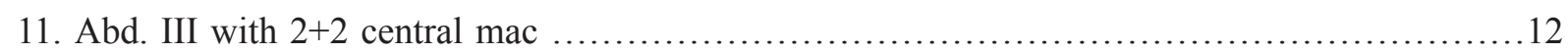

- Abd. III with $3+3$ central mac ....................................................... 13

12. Abd. III with $3+3$ lateral mac ................................... baoshanensis sp. nov.

- Abd. III with 4+4 lateral mac ................................ guangxiensis Shi \& Chen, 2004 
13. Scales absent on antennae and manubrium ......W. yiningensis Zhang, Chen \& Deharveng, 2011

- Scales present on antennae and manubrium .............................................. 14

14. Abd. II-III with blue transverse bands ........................... zhaotongensis sp. nov.

- Abd. II-III with scattered blue pigment and irregular patches ......W. shi Pan, Zhang \& Chen, 2006

\section{Discussion}

Entomobryidae is the largest family of Collembola in China and about 140 species have been reported. Willowsia, a genus of the subfamily Entomobryinae which includes about 120 Chinese species, contains relatively few species with only 13 species reported from China prior to this study. Two new species of the genus were added here: $W$. baoshanensis sp. nov. and $W$. zhaotongensis sp. nov., and it is the first report of the genus from Yunnan Province, southwest China. There are different climate zones in Yunnan, including northern tropical, subtropical, temperate and highland climate zones suggesting a rich biodiversity might be expected. However, due to the lack of research conducted in the area so far, there are only a few records of Collembola from the province.

\section{Acknowledgements}

We thank Xinnan Jiang and Fudong Ding who collected the specimens.

\section{References}

Gisin H. 1967. Espèces nouvelles et lignées évolutives de Pseudosinella endogés. Memórias e Estudos do Museu Zoológico da Universidade de Coimbra 301: 5-25.

Jordana R. \& Baquero E. 2005. A proposal of characters for taxonomic identification of Entomobrya species (Collembola, Entomobryomorpha), with description of a new species. Abhandlungen und Berichte des Naturkundemuseums Goerlitz 76 (2): 117-134.

Mari-Mutt J.A. 1986. Puerto Rican species of Lepidocyrtus and Pseudosinella (Collembola: Entomobryidae). Caribbean Journal of Science 22 (1-2): 1-48.

Pan Z.X. \& Zhang F. 2016. Contribution to the Willowsia species having body scales of long basal rib type: four new species and a redescription of W. qui (Collembola: Entomobryidae). European Journal of Taxonomy 245: 1-25. https://doi.org/10.5852/ejt.2016.245

Pan J.L., Zhang F. \& Chen J.X. 2006. A new Chinese species of the genus Willowsia from Tibet (Collembola: Entomobryidae). Journal of the Kansas Entomological Society 79 (3): 261-266. https:// doi.org/10.2317/0511.22.1

Schäffer C. 1896. Die Collembolen der Umgebung von Hamburg und benachtbaren Gebiete. Mittheilungen aus dem Naturhistorischen Museum 13: 147-216. Available from http://biodiversitylibrary.org/ page/29521571 [accessed 15 Mar. 2017]

Shi X. \& Chen J.X. 2004. The genus Willowsia (Collembola: Entomobryidae) and species from China. Entomotaxonomia 26 (4): 241-248.

Shoebotham J.W. 1917. Notes on the Collembola, part 4. The classification of the Collembola; with a list of genera known to occur in the British Isles. Annals and Magazine of Natural History series 8, 19: 425-436. Available from http://biodiversitylibrary.org/page/22130994 [accessed 15 Mar. 1017]

Szeptycki A. 1979. Morpho-Systematic Studies on Collembola. IV. Chaetotaxy of the Entomobryidae and its Phylogenetical Significance. Polska Akademia Nauk, Kraków. 
Zhang F., Chen J.X. \& Deharveng L. 2011. New insight into the systematics of the Willowsia complex (Collembola: Entomobryidae). Annales de la Société Entomologique de France 47 (1-2): 1-20. https:// doi.org/10.1080/00379271.2011.10697692

Manuscript received: 9 May 2016

Manuscript accepted: 24 August 2016

Published on: 20 April 2017

Topic editor: Gavin Broad

Desk editor: Kristiaan Hoedemakers

Printed versions of all papers are also deposited in the libraries of the institutes that are members of the EJT consortium: Muséum national d'Histoire naturelle, Paris, France; Botanic Garden Meise, Belgium; Royal Museum for Central Africa, Tervuren, Belgium; Natural History Museum, London, United Kingdom; Royal Belgian Institute of Natural Sciences, Brussels, Belgium; Natural History Museum of Denmark, Copenhagen, Denmark; Naturalis Biodiversity Center, Leiden, the Netherlands. 